\title{
ARQUITECTURA DE UN SISTEMA DE MEDICIÓN DE BIOPARÁMETROS INTEGRANDO SEÑALES INERCIALES- MAGNÉTICAS Y ELECTROMIOGRÁFICAS
}

\author{
Mauro Callejas-Cuervo', Manuel A. Vélez-Guerrero², Wilson Javier Pérez Holguín ${ }^{3}$
}

1PhD en Energía y Control de Procesos. Grupo de Investigación GIS. mauro.callejas@uptc.edu.co 2Magister en ingeniería. Grupo de Investigación GIS. manuel.velez@uptc.edu.co ${ }^{3} \mathrm{PhD}$ en ingeniería. Grupo de Investigación GIRA. wilson.perez@uptc.edu.co 1,2,3Universidad Pedagógica y Tecnológica de Colombia. Tunja-Colombia

\section{RESUMEN}

Este trabajo presenta una arquitectura para la medición e integración de bioparámetros basado en unidades de procesamiento de movimiento inercial-magnético (MPUs) y electromiografía (EMG). Derivado de la arquitectura propuesta, se logró desarrollar un dispositivo llamado Imocap, el cual reúne y utiliza las mejores características de la tecnología MPU + EMG para realizar una medición completa en el segmento de brazo y antebrazo en el cuerpo humano. Se presenta en primer lugar la revisión bibliográfica de los métodos y herramientas para la captura del movimiento biomecánico, seguido de las técnicas y aplicaciones de la recolección de bioparámetros. Finalmente, se muestra la arquitectura y la descripción del sistema Imocap, algunas aplicaciones y discusión. Como trabajo futuro, Imocap tiene como objetivo proporcionar la información necesaria en un sistema de control electrónico para una plataforma de rehabilitación basada en exoesqueletos robóticos.

Palabras clave: Rehabilitación; miembros superiores; captura de movimiento; electromiografía; fusión de datos.

Recibido: 31 de octubre de 2018. Aceptado: 29 de Noviembre de 2018

Received: October 31th, 2018. Accepted: November 29th, 2018

\section{BIOPARAMETER MEASUREMENT SYSTEM ARCHITECTURE INTEGRATING INERTIAL-MAGNETIC AND ELECTROMYOGRAPHIC SIGNALS}

\begin{abstract}
This paper presents an architecture for the measurement and integration of bioparameters based on inertialmagnetic motion processing units (MPUs) and electromyography (EMG). Derived from the proposed architecture, a device called Imocap was developed. Imocap gathers and uses the edge-cutting features of $M P U$ + EMG technology to perform a complete measurement in the arm and forearm segment in the human body. The literature review of the methods and tools for the biomechanical movement capture is presented in first place, followed by the techniques and applications of the bioparameters collection. Finally, this paper shows the proposed architecture and the description of the Imocap system, some applications and discussion. As future work, Imocap aims to provide the necessary information in an electronic control system for a rehabilitation platform based on robotic exoskeletons.
\end{abstract}

Keywords: Upper-limbs; rehabilitation; motion capture; electromyography; data fusion.

Cómo citar este artículo: M. Callejas Cuervo, M. A. Vélez Guerrero, W. J. Pérez Holguín, "Arquitectura de un sistema de medición de bioparámetros integrando señales inerciales-magnéticas y electromiográficas", Revista Politécnica, vol. 14, no. 27 pp.93-102, 2018. https://doi.org/10.33571/rpolitec.v14n27a9 


\section{INTRODUCCIÓN}

La discapacidad motora, en cualquiera de sus formas, es parte de la condición humana: casi todas las personas sufrirán algún tipo de discapacidad temporal o permanente en algún momento de sus vidas, y los que llegan a la vejez experimentarán crecientes dificultades en el funcionamiento del sistema motor [1]. En Colombia, según el Informe de la Sala Nacional Situacional de Personas con Discapacidad [2], hay un total de 1'265.328 personas con discapacidad, donde la afección principal se produce en los movimientos del cuerpo, manos, brazos y piernas, con $33.8 \%$ de la población total afectada.

Con el advenimiento de nuevos sistemas de rehabilitación debido al auge tecnológico, la medicina y ciencias de la salud han podido extender sus alcances. En la actualidad, sistemas tecnológicos especializados de medición son utilizados en áreas como el diagnóstico clínico [3], así como el desarrollo de dispositivos de rehabilitación activa empleados en técnicas de fisioterapia para las extremidades del cuerpo humano [4], propiciando ambientes favorables para la recuperación de los trastornos motores.

Siendo este el panorama de la investigación, la propuesta aquí presentada consiste en el diseño de una arquitectura para realizar mediciones de bioparámetros (señales físicas, fisiológicas o químicas producidas por un organismo) en las extremidades superiores del cuerpo humano, integrando específicamente señales inercialmagnéticas y electromiográficas.

El propósito del desarrollo de esta arquitectura recae en la creación de sistemas tecnológicos robustos que permitan una adecuada medición de bioparámetros, y la utilización de dichas señales en procesos de diagnóstico y rehabilitación clínica. En cuanto a ingeniería, la investigación está centrada en el desarrollo de un sistema de recolección bioparamétrica, proponiendo un dispositivo hardware de medida e integración adecuado. Dentro del área médica, el enfoque está puesto en la caracterización y recolección de los bioparámetros, su análisis y caracterización para poder ser procesados en el sistema tecnológico.

A través del desarrollo del presente trabajo, se pretende demostrar que la inclusión de dispositivos de medición precisos, confiables y seguros trae beneficios en la asistencia de los procesos de rehabilitación de los miembros superiores, reduciendo el tiempo de recuperación de los individuos y equipando a los profesionales médicos y fisioterapeutas con herramientas tecnológicas que les permitan hacer su trabajo de manera más oportuna y efectiva.

Se presenta inicialmente una revisión cronológica de la literatura, identificando las diferentes tecnologías utilizadas, incluyendo la información cinemática extraída de las Unidades Procesadoras de Movimiento (MPU) y el uso de señales electromiográficas (EMG). Posteriormente se expone una propuesta de arquitectura para el desarrollo de un sistema de medición de bioparámetros. Finalmente se plantea la discusión y conclusión derivada de la investigación.

\section{REVISIÓN DE LITERATURA}

El objetivo es presentar una revisión exhaustiva de la bibliografía sobre técnicas y sistemas de recolección de bioparámetros, como los potenciales de movimiento y la activación muscular utilizados como señales de control en los sistemas de rehabilitación asistida. Se enfatiza en aquellos desarrollos que aportan en el proceso de rehabilitación de la función motora de las extremidades superiores del cuerpo humano con exoesqueletos robóticos y prótesis activas.

\subsection{MÉTODO}

La metodología de revisión bibliográfica comprende los artículos: 1) publicados desde el año 2015, 2) que incluyen procedimientos de recolección de información de bioparámetros, 3) centrados en el uso de diferentes tecnologías, y 4) que opcionalmente empleen dispositivos exoesqueléticos para fines de rehabilitación.

De 423 artículos encontrados con las características mencionadas, el $48 \%$ se obtuvieron de Scopus, el $23 \%$ en IEEE Xplore Digital Library, el $16 \%$ en ScienceDirect y el $11 \%$ en PubMed, junto con el $2 \%$ de otras fuentes. Se eliminaron los documentos duplicados o menos relacionados, reduciendo la muestra a 247 documentos. Finalmente, los documentos se clasificaron según las herramientas tecnológicas utilizadas en el proceso de recolección de la información, el proceso de rehabilitación y el papel de los sistemas exoesqueléticos activos de rehabilitación. 


\subsection{SISTEMAS DE RECOLECCIÓN DE BIOPARÁMETROS BASADOS EN EMG.}

En cuanto a las herramientas y técnicas de medición de bioparámetros para el control de los sistemas exoesqueléticos robóticos, las contribuciones de Chakarov, et al. [5] sobresalen por el diseño de un exoesqueleto para las extremidades superiores en aplicación de la interacción humana con las tareas virtuales de un entorno para rehabilitación.

El equipo de trabajo de Zhichuan, et al. [6] desarrolló un sistema de control mioeléctrico para el manejo de un exoesqueleto robótico de miembro superior de acuerdo a la intención del movimiento del usuario en tiempo real. En [7] se muestra que el uso de bioparámetros es efectivo para el control de un exoesqueleto de mano, específicamente utilizando técnicas de reconocimiento de patrones en EMG. Los resultados muestran un gran potencial para aplicar un control basado en bioparámetros buscando mejorar la función de la mano después de lesiones neurológicas.

Otros trabajos se centran en el procesamiento de señales EMG realizando una estimación continua de la amplitud articular [8]. Se destaca el uso de sistemas de electromiografía superficial (sEMG) en el trabajo de Lambelet [9] para el control de un exoesqueleto robótico de 1 Grado de Libertad (DOF). El objetivo es proporcionar un dispositivo de entrenamiento y rehabilitación que mejore la actividad de los músculos extensores de la muñeca, combinando la fuerza y el control basado en sEMG en una estrategia de asistencia y apoyo.

De manera similar, Tiboni [10] implementó una estrategia de control para un exoesqueleto robótico basado en EMG. Este sistema se proyecta para la rehabilitación del codo utilizando un exoesqueleto activo. Adicionalmente, mediante el control de un exoesqueleto robótico activo en [11], se evidenció que el uso de un control basado en señales EMG es efectivo incluso si se combina con otro tipo de detección de intención de movimiento. Finalmente, Hwang y su equipo [12] analizaron el par y la función muscular con gran precisión mientras realizaban ejercicios de rehabilitación con un exoesqueleto robótico, demostrando la validez de su aplicación.

\subsection{INTEGRACIÓN DE TECNOLOGÍA PARA LA RECOLECCIÓN DE BIOPARÁMETROS.}

Dentro de las nuevas tecnologías utilizadas en la medición de parámetros enfocados al uso en exoesqueletos, se encuentran principalmente sistemas de medición electromiográfica, electroencefalográfica (EEG), y un menor número de sensores de otra naturaleza. Sarasola-Sanz [13] describe cómo los sistemas basados en EMG-EEG híbridos (llamados interfaces híbridas de electroencefalografía hBMls) son capaces de generar mejores resultados aplicados en sesiones de rehabilitación con exoesqueleto activo, logrando detectar y controlar sistemas exoesqueléticos de 7DOF.

En el trabajo de Qingsong, et al. [14] se fusionan parámetros de biodetección EMG y de aceleración que proveen información sobre la intención de movimiento de un paciente. Los resultados demuestran que la clasificación basada en fusión de datos supera a la clasificación realizada con señales individuales, donde la fusión alcanza una precisión de reconocimiento del $95 \%$ o superior, lo que demuestra la validez del método propuesto.

Mazumder presenta en [15] una fusión de datos entre unidades de medida inercial y EMG. Centrado en la misma idea, el trabajo propuesto por Joukov [16] proporciona información útil sobre la interacción de los sistemas de medición, los sensores inerciales-magnéticos y los exoesqueletos. Se destaca que la información recogida en el sistema propuesto muestra que es posible fusionar la información de diferentes sistemas para obtener el control deseado en los exoesqueletos activos.

Finalmente, se han construido otros tipos de interfaces hombre-máquina basadas en diferentes métodos de recolección de información, como el caso del control de los exoesqueletos activos mediante sistemas de resonancia magnética [17], mediante encefalografía [18] o mediante una combinación de los dos sistemas, como el caso de Tobar [19], en el que el punto de convergencia entre todas estas tecnologías es el desarrollo de colectores de información eficientes para su uso en aplicaciones médicas, más concretamente en el control activo de los exoesqueleto robóticos que se enmarcan en un contexto de rehabilitación. 


\subsection{SISTEMAS EXOESQUELÉTICOS.}

Los estudios enfocados a la caracterización de sistemas mecatrónicos para la rehabilitación incluyen el aporte de H. Zeng-Gang, et al. [20], donde se propone el diseño de robots de rehabilitación y sistemas inteligentes de asistencia que "ofrecen soluciones potenciales a las personas mayores y discapacitadas con métodos eficaces de cuidado, asistencia y rehabilitación". Otros estudios simulan características robóticas que proporcionan mejoras en pacientes con sistemas locomotores deficientes [21] o la creación de técnicas de control especializadas [22-23] para generar exoesqueletos más robustos con fines de rehabilitación.

Específicamente para los miembros superiores del cuerpo humano, se han diseñado e incorporado sistemas de asistencia mecánica para la rehabilitación. Por ejemplo, Yupeng [24] ha desarrollado un robot de exoesqueleto de miembros superiores Ilamado IntelliArm, asistiendo los movimientos del hombro, el codo y la muñeca para apoyar procesos de pre evaluación multiarticular.

En el trabajo de Keller, et al. [25], se desarrolló Charmin, un exoesqueleto que ayuda a los niños con parálisis cerebral a realizar movimientos de brazos. La eficacia de Charmin se demostró a través de experimentos con supervivientes de accidentes cerebrovasculares y sujetos sanos. Kooren, et al. [26] propone un soporte activo del brazo que aumenta las capacidades de movimiento residual de las personas con distrofia muscular de Duchenne.

El avance comercial de los sistemas exoesqueléticos activos es numeroso para diferentes propósitos en el campo médico, como por ejemplo el diseño de planes de asistencia para centros de rehabilitación especializados, incluyendo el exoesqueleto LOPES [27], Lokomat [28], y los entrenadores del movimiento de marcha CAPIO y HAL5 [29]. Algunos de los productos exoesqueléticos ofrecen asistencia a personas parapléjicas, por ejemplo, Ekso Bionics eLEGS [30], un exoesqueleto robótico biónico que puede ser colocado como un dispositivo portátil para mejorar la fuerza, movilidad y resistencia de los miembros superiores de soldados y personas parapléjicas.

\subsection{RESULTADOS DE LA REVISIÓN.}

Como lo muestra la revisión de la literatura, no existe una propuesta sólida que proponga la arquitectura de un dispositivo de captura de información cinemática y electromiográfica para el segmento de brazo y antebrazo enfocado en procesos de rehabilitación, lo que da cabida a la presente investigación.

Complementario al desarrollo de sistemas de medición más robustos y confiables, algunos autores tratan el uso de dispositivos exoesqueléticos robóticos para diferentes tipos de rehabilitación, aunque no hay mayor énfasis en su uso como un apoyo decisivo en la resolución de problemas motores en las extremidades superiores de los pacientes.

La incursión de sistemas electrónicos de medición también puede ser considerada propicia en el área específica de la medicina y la salud, pensando en mecanismos que puedan contribuir activamente a la mejora de los pacientes. La proyección que este desarrollo pretende dar al uso de la tecnología es de gran relevancia porque contribuye a mejorar la calidad de vida de las personas en general, y ofrece un conocimiento integral en muchos campos, mostrando el potencial investigador que el presente tema merece.

\section{DESCRIPCIÓN DE LA ARQUITECTURA}

Teniendo en cuenta los sistemas propuestos anteriormente por otros equipos de trabajo, el creciente interés por el análisis objetivo de diferentes parámetros del cuerpo humano, así como las diversas aplicaciones en áreas de ingeniería, deporte o ciencias de la salud, nos ha impulsado a desarrollar sistemas portátiles de captura de bioparámetros, aprovechando el conocimiento en dispositivos biomédicos, embebidos y la tecnología de desarrollo.

La siguiente sección describe la arquitectura de un sistema de medición de bioparámetros, el cual integra señales inerciales-magnéticas y electromiográficas. Esta arquitectura permite una evaluación en el segmento del brazo y del antebrazo usando dispositivos de distinta tecnología. Se profundiza en la descripción de los sub-sistemas que componen la arquitectura. 


\subsection{PROCESAMIENTO CENTRAL: IMOCAP}

El diseño del sistema central de procesamiento parte de la conceptualización del propio sistema: los sensores que captan el movimiento sobre los segmentos del antebrazo y brazo, incluyendo los electrodos para la captura de la actividad electromiográfica muscular, se conectan alámbricamente a una unidad central de proceso llamada Imocap.

Este sistema, sintetizado en la Fig.1., se compone de un microprocesador System-on-Chip (SoC), encargado del funcionamiento principal de Imocap, procesamiento de la información y coordinación de las tareas en ejecución; interfaces de conexión de periféricos que permiten la reconfiguración del dispositivo para múltiples aplicaciones; unidad de almacenamiento masivo, dando soporte a recolección de datos de manera local; comunicación inalámbrica vía Wi-Fi con diversos modos de operación; y un sistema de gestión eléctrica que otorga versatilidad y mayor capacidad de autonomía.

Inicialmente, el uso de Imocap supone una ventaja frente a otros sistemas: su diseño compacto y embebido permite integrar todas las partes funcionales para recolectar, procesar, almacenar y transmitir los bioparámetros sin emplear un gran tamaño.

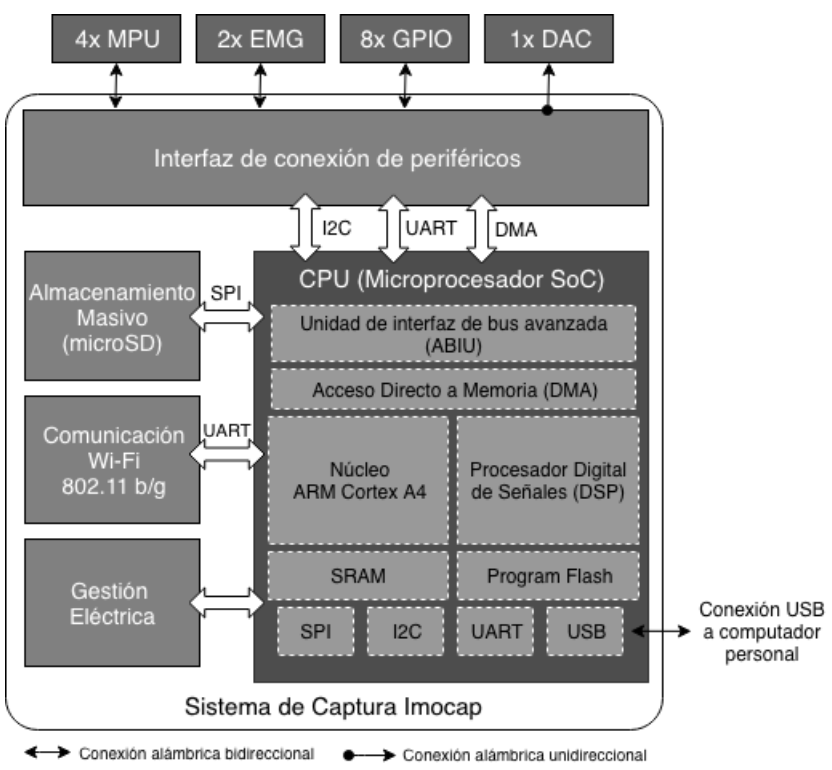

Fig.1. Sinopsis centralizada del sistema de captura embebido Imocap.
Independientemente de las interfaces para captura de movimiento biomecánico (MPUs) y electromiografía (EMG) que serán analizados posteriormente, posee conectores para la adición de periféricos a través de 8 canales de propósito general GPIO (4 analógicos de entrada 12-bit ADC, 4 digitales bidireccionales).

Además, cuenta con canal dedicado para generación de señales analógicas (16-bit DAC) controlado por la interfaz de acceso directo a memoria (DMA) y una conexión USB para transmisión de datos, reprogramación del firmware embebido y recarga de batería. En la Fig.2. (a) se muestra el hardware interno del sistema Imocap, mientras que en (b) se muestra el diseño final del sistema con una carcasa impresa en 3D.
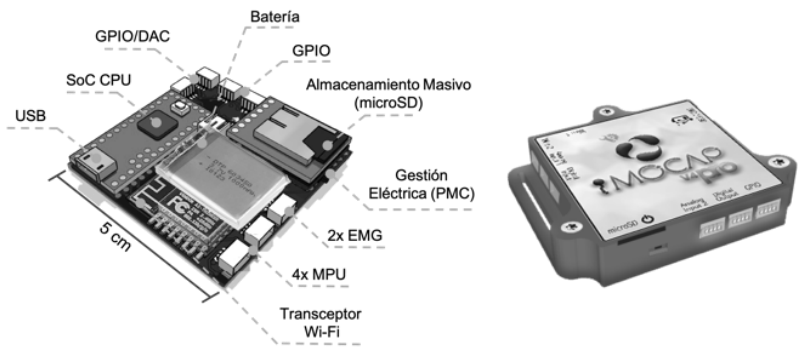

Fig.2. (a) Hardware interno Imocap (b) Sistema Imocap con carcasa impresa en 3D.

Cabe destacar que el microprocesador embebido en conjunto con características del firmware, posibilita el procesamiento de los bioparámetros adquiridos incluyendo fusión de datos, aplicación de filtrado digital, entre otros procesos.

\subsection{SISTEMA DE CAPTURA DE MOVIMIENTO BIOMECÁNICO}

Imocap soporta simultáneamente hasta 4 Unidades de Procesamiento de Movimiento (MPUs) basadas en tecnología inercial-magnética. La captura de movimiento biomecánico puede realizarse paralelamente a otros procesos de Imocap, como la captura EMG o transmisión de datos.

La interfaz de conexión de periféricos detecta automáticamente el tipo de MPU utilizado y reconfigura los parámetros internos del sistema para adecuarse a los sensores conectados. Se utilizaron sensores MPU comunes que utilizan el estándar de comunicación Inter-Integrated Circuit (I2C), específicamente Invensense MPU-9250. 
En la Fig.3. (a) se presenta una de las unidades MPU-9250 usadas en el sistema Imocap junto a una escala de referencia, y (b) el sistema de conexión de múltiples unidades MPU a Imocap.

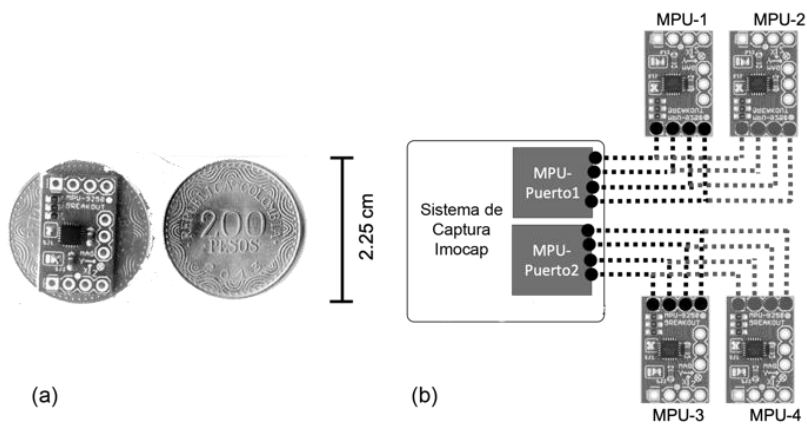

Fig.3. (a) MPU-9250 junto a una escala de referencia. (b) múltiples unidades MPU a Imocap.

La velocidad de muestreo de cada MPU depende de las características propias de cada uno de los sensores, siendo predefinida una tasa de 30 muestras/segundo. Para el caso de MPU-9250, Imocap soporta hasta 90 muestras/segundo por cada una de las unidades conectadas al sistema. Teniendo como referente algunos de los sistemas presentados en la revisión de literatura, la velocidad de muestreo suministrada por Imocap se encuentra por encima de la velocidad de muestreo promedio.

Se presenta en la Tabla 1 los múltiples dispositivos MPU de tecnología inercial-magnética soportados por Imocap con sus respectivas tasas de muestreo máximas. La gran variedad de dispositivos soportados por el sistema de captura permite su uso en diferentes aplicaciones de recolección de movimiento biomecánico, desvaneciendo también los límites de interoperabilidad entre dispositivos de tecnología similar que se encuentran en diferentes familias.

Tabla 1. MPUs soportados por Imocap.

\begin{tabular}{lc}
\hline Dispositivo & $\begin{array}{c}\text { Muestreo Máximo } \\
\text { [muestras/s] }\end{array}$ \\
\hline Invensense MPU-9255 & 100 \\
Invensense MPU-9250 & 90 \\
Invensense MPU-9150 & 60 \\
STM L3GD20H + LSM303D & 120 \\
STM LSM9DS0 & 190 \\
STM LSM9DS1 & 380 \\
Bosch BMX055-BNX055 & 400 \\
\hline
\end{tabular}

\subsection{SISTEMA DE CAPTURA DE INFORMACIÓN ELECTROMIOGRÁFICA}

Uno de los elementos diferenciadores de la arquitectura propuesta es la posibilidad de recolección y procesamiento de señales electromiográficas (EMG). Esto permite que la información de movimiento recogida a través de las MPUs se complemente con registros de actividad mioeléctrica, abriendo un amplio abanico de posibles análisis y proporcionando así nuevas métricas de apoyo, como la determinación de la fatiga muscular, torque, entre otros.

La recolección de señales EMG requiere el uso de hardware externo para el acondicionamiento de señales, en donde una amplia variedad de dispositivos comerciales están soportados. Por defecto, la velocidad de muestreo de las señales EMG es la misma establecida para las unidades MPU, garantizando así la sincronización de los datos y la apropiada fusión de la información.

En la Fig.3. (a) se presenta un sistema de acondicionamiento EMG MyoWare, y en (b) el sistema de conexión de múltiples unidades EMG a Imocap.

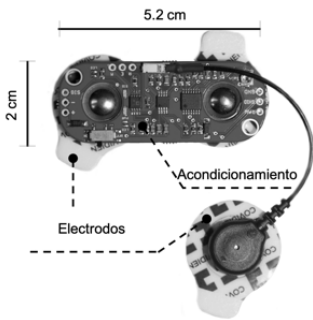

(a)

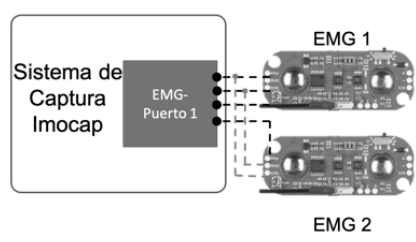

(b)
Fig.4. (a) Acondicionamiento EMG MyoWare. (b) múltiples unidades EMG conectadas a Imocap.

Complementando la fusión de datos de MPU+EMG, se desarrollaron algoritmos que permiten la detección de gestos o patrones en las señales electromiográficas recolectadas, acelerando el desarrollo de aplicaciones que requieren este tipo de información extraída directamente del proceso de captura.

\subsection{PROCESAMIENTO DIGITAL}

El sistema Imocap contiene algoritmos especializados para el tratamiento de la información de los bioparámetros recolectados. 
Los algoritmos diseñados hacen uso del procesador digital de señales (DSP) embebido, y están diseñados para ofrecer mejoras en cuanto a la calidad de la información recolectada, proporcionando de manera adecuada la información procesada y disponible para cualquier aplicación que lo requiera.

En la Tabla 2 se muestra un resumen de los algoritmos disponibles dentro del sistema de captura y que enriquecen la funcionalidad de la plataforma. Todo el procesamiento digital se realiza dentro del hardware de Imocap.

Tabla 2. Algoritmos de Procesamiento en Imocap.

\begin{tabular}{|c|c|c|c|}
\hline Algoritmo & $\begin{array}{l}\text { Aplicable } \\
\text { a MPU }\end{array}$ & $\begin{array}{c}\text { Aplicable a } \\
\text { EMG }\end{array}$ & $\begin{array}{c}\text { Aplicable } \\
\text { a GPIO }\end{array}$ \\
\hline $\begin{array}{l}\text { Filtro de Kalman } \\
\text { Extendido }\end{array}$ & $\checkmark$ & $\checkmark$ & $x$ \\
\hline $\begin{array}{l}\text { Filtro de Kalman } \\
\text { Unscented }\end{array}$ & $\checkmark$ & $\checkmark$ & $x$ \\
\hline Filtro LPF & $\checkmark$ & $\checkmark$ & $\checkmark$ \\
\hline Filtro HPF & $\checkmark$ & $\checkmark$ & $\checkmark$ \\
\hline $\begin{array}{l}\text { Fusión de datos } \\
\text { (Partículas / Kalman) }\end{array}$ & $\checkmark$ & $\checkmark$ & $\checkmark$ \\
\hline RMSE & $\checkmark$ & $\checkmark$ & $\checkmark$ \\
\hline Media & $\checkmark$ & $\checkmark$ & $\checkmark$ \\
\hline Media Cuadrática & $\checkmark$ & $\checkmark$ & $\checkmark$ \\
\hline Calibración & $\checkmark$ & $\checkmark$ & $x$ \\
\hline $\begin{array}{l}\text { Detección de } \\
\text { Gestos }\end{array}$ & $x$ & $\checkmark$ & $x$ \\
\hline
\end{tabular}

El sistema de calibración que Imocap utiliza en MPUs y EMGs depende del sensor conectado al sistema, guiando al usuario a través de pasos que permiten la correcta adecuación de parámetros. Se asegura que tanto las señales de movimiento biomecánico, así como las variaciones mioeléctricas sean capturadas en condiciones óptimas de línea base, reduciendo el error asociado.

\subsection{OTROS SUBSISTEMAS INTEGRADOS}

El sistema de captura de bioparámetros Imocap contiene dos subsistemas adicionales que permiten explotar a mayor capacidad las funciones de captura de movimiento y electromiografía.

Se proporciona un transceptor Wi-Fi $802.11 \mathrm{~b} / \mathrm{g}$ el cual permite realizar envío de la información procesada hacia un sistema receptor principal, como puede ser el caso de un computador personal.
Además, Imocap ofrece diferentes modos de conexión inalámbrica, pudiendo actuar en modo "malla de interconexión" entre múltiples sistemas Imocap, donde el nodo principal transmite o recolecta la información de los nodos esclavos, extendiendo los límites de captura de movimiento a múltiples dispositivos en simultáneo. Complementario a la transmisión de datos de forma inalámbrica, Imocap hace uso de un sistema de almacenamiento masivo basado en microSD, lo cual permite la recolección local de grandes volúmenes de información. Debido al uso de un formato estándar, los datos recolectados por Imocap pueden ser luego analizados a través de diferentes plataformas libres o comerciales.

Finalmente, se incluye un sistema de gestión de energía PMC (Power Management Controller) el cual regula el consumo de energía con el fin de prolongar la autonomía de la batería. Con la utilización del PMC, Imocap puede recolectar señales a máxima capacidad (4 MPUs + 2 EMG + Comunicación $\mathrm{Wi}-\mathrm{Fi}$ + Almacenamiento SD) con autonomía suficiente para 2 horas continuas. Esto brinda una gran ventaja frente a sistemas de recolección de datos no embebidos, ya que permite la portabilidad del sistema sin sacrificar rendimiento o funcionalidad.

\section{APLICACIONES}

El desarrollo de una arquitectura completa de medición, centrada en la recolección, procesamiento y transmisión de información bioparamétrica, puede cubrir un amplio espectro de aplicaciones. Dentro del área médica y de ciencias de la salud, la evaluación apropiada de bioparámetros se ha discutido con anterioridad, destacando los procesos de diagnóstico asistido por tecnología.

Se han realizado pruebas satisfactorias usando Imocap, las cuales están destinadas a la determinación de amplitudes articulares por medio del sistema inercial-magnético [31], así como el rastreo de los movimientos del segmento del brazo y antebrazo para procesos de rehabilitación [32]. En el mismo sentido, se ha utilizado esta arquitectura y específicamente la información EMG para el reconocimiento de patrones [33], análisis preliminares de fatiga muscular y el desarrollo de protocolos de prueba y aplicación de señales EMG en contextos académicos y de laboratorio, como se muestra en la Figura 5. 
Además, Imocap se utilizó satisfactoriamente en la evaluación de la posición del segmento inferior del tronco, permitiendo la determinación de parámetros relacionados con el equilibrio. Otros usos de este sistema se encuentran en el área de deportes y entretenimiento, donde es frecuente el uso de sistemas de medición biomecánica como herramienta de asistencia o digitalización de la información.

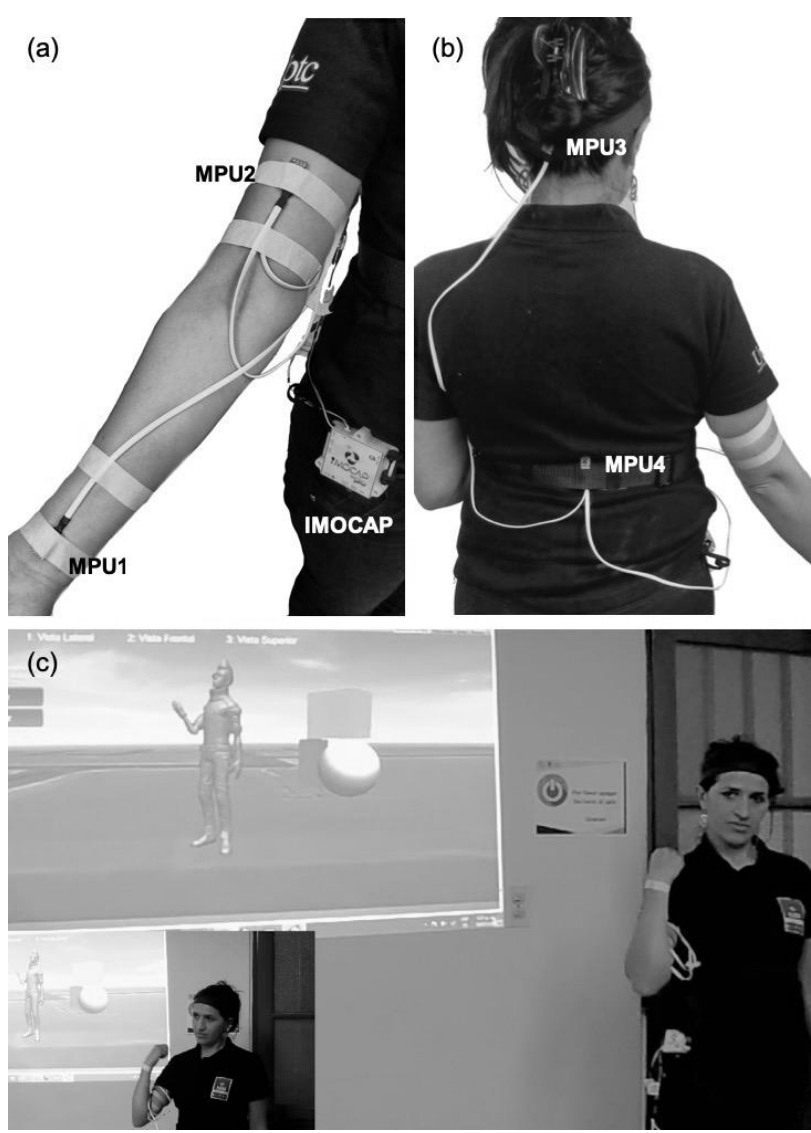

Fig.5. Uso del sistema Imocap como herramienta de análisis y recolección de información biomecánica, en interacción con objetos virtuales y animación. (a) Vista frontal. (b) Vista posterior. (c) Aplicación.

\section{DISCUSIÓN}

Teniendo como referente los trabajos de Mazumder [15] y Joukov [16], la implementación de algoritmos de procesamiento dedicados en el sistema Imocap mejoran la adquisición y procesamiento de los bioparámetros, ubicando a la arquitectura propuesta como un candidato potencial para ser usado en aplicaciones de control. Si se compara la arquitectura con trabajos precursores como el caso de Qingsong, et al. [14], resalta notoriamente la fusión de datos entre sistemas de medición de movimiento a través de MPU y señales electromiográficas, ya que las señales provenientes de MPU son más completas al integrar magnetómetro y giróscopo.

Desde el punto de vista del tratamiento de señales EMG, Imocap presenta la posibilidad de análisis, captura y transmisión de la información. Difiere de las investigaciones presentadas por Chakarov [5] o Tang [6], ya que no se encuentra enmarcada dentro de un contexto orientado al desarrollo de sistemas de control, abriendo así una posibilidad más directa en miras de ofrecer un sistema que pueda ser usado en asistencia médica o diagnóstica.

Como un punto de valor agregado a la arquitectura, se resalta que el costo total de desarrollo total está por debajo de los costos asociados al desarrollo de otras tecnologías similares, lo que puede llegar a promover su uso. Así mismo, al ofrecer portabilidad, facilidad y robustez en la recolección y análisis de los bioparámetros cinemáticos y electromiográficos, la tecnología utilizada podría atraer un mayor público.

\section{CONCLUSIONES}

Se considera que la contribución central de esta investigación se da en términos de la proposición de una arquitectura para la medición de bioparámetros, cuyo uso está enfocado, pero no limitado exclusivamente, a proveer mejores resultados en aplicaciones dentro del área de la medicina y ciencias de la salud.

A través del diseño, construcción y verificación del funcionamiento del sistema Imocap se comprobó que la integración de diversas tecnologías provee resultados prometedores independientemente del tipo de aplicación seleccionada, validando así la arquitectura presentada inicialmente. En cuanto a la usabilidad del sistema propuesto, se resalta la inclusión de algoritmos digitales de procesamiento, brindando mayor precisión y confiabilidad en la información. Adicionalmente, debido a la transferencia de datos a través de Wi-Fi o la posibilidad de almacenamiento local, Imocap no requiere cables para la comunicación de la información biomecánica.

La autonomía de la batería, que ronda las 2 horas de uso continuo en máxima capacidad y la 
posibilidad de interoperación de diversos sensores con la plataforma, convierten al sistema desarrollado en un punto de partida crucial para el desarrollo de mejores sistemas integrados de medición de bioparámetros.

Se evidenciaron algunas desventajas con el uso de este sistema, dentro del cual se destacan las dimensiones físicas de la versión equipada con carcasa impresa en 3D respecto a otros desarrollos actuales, entre otros aspectos del diseño técnico.

Como trabajo futuro se planea la implementación de un sistema Imocap de dimensiones físicas reducidas que facilite su posicionamiento en el cuerpo humano. Se espera usar la arquitectura de medición aquí presentada para el diseño e implementación de sistemas de control robustos, dedicados a la operación de sistemas de rehabilitación basados en exoesqueletos robóticos. Se considera como un aporte significativo sentar las bases para mejorar la implementación del control electrónico de los sistemas robóticos exoesqueléticos enfocados en rehabilitación.

\section{REFERENCIAS}

[1] V. Feigin, G., et al. Global burden of stroke and risk factors in 188 countries, during 1990-2013: a systematic analysis for the Global Burden of Disease Study 2013, The Lancet Neurology, vol. 15, 913-924, 2016.

[2] Ministerio de Salud y Protección Social de Colombia. Sala Situacional de Personas con Discapacidad (PCD), 2016.

[3] D. Hill, C. Holloway, D. Morgado Ramirez, P. Smitham y Y. Pappas. What are User Perspectives of Exoskeleton Technology? A Literature Review, Intl. Journal of Tech. Assessment in Health Care, vol. 33, 160-167, 2017.

[4] A. Stephenson y J. Stephens. An exploration of physiotherapists' experiences of robotic therapy in upper limb rehabilitation within a stroke rehabilitation centre, Disability and Rehabilitation: Assistive Technology, vol. 13, 245-252, 2018.

[5] D. Chakarov, I. Veneva, M. Tsveov y T. Tiankov. New Exoskeleton Arm Concept Design and Actuation for Haptic Interaction With Virtual Objects, Journal of Theo. and App. Mech., vol. 44, 3-14, 2015.
[6] Z. Tang, K. Zhang, S. Sun, Z. Gao, L. Zhang, y Z. Yang. An Upper-Limb Power-Assist Exoskeleton Using Proportional Myoelectric Control, Sensors, 6677-6694, 2015.

[7] Z. Lu, X. Chen, X. Zhang, K.-Y. Tong, y P. Zhou. Real-Time Control of an Exoskeleton Hand Robot with Myoelectric Pattern Recognition, Int. J. Neural Syst., vol. 27, 2017.

[8] F. Xiao, Y. Wang, Y. Gao, Y. Zhu, y J. Zhao. Continuous estimation of joint angle from electromyography using multiple time-delayed features and random forests, Biomed. Signal Process. Control, vol. 39, 303-311, 2018.

[9] C. Lambelet, M. Lyu, D. Woolley, R. Gassert, y N. Wenderoth. The eWrist: A wearable wrist exoskeleton with sEMG-based force control for stroke rehabilitation, en International Conference on Rehabilitation Robotics, 726-733, 2017.

[10] M. Tiboni, et al. ERRSE: Elbow robotic rehabilitation system with an EMG-based force control, 26th International Conference on Robotics in Alpe-Adria-Danube Region, RAAD 2017, vol. 49, 892-900, 2018.

[11] J. R. Koller, C. David Remy, y D. P. Ferris. Comparing neural control and mechanically intrinsic control of powered ankle exoskeletons, en 2017 International Conference on Rehabilitation Robotics, 294-299, 2017.

[12] B. Hwang y D. Jeon. Estimation of the User's Muscular Torque for an Over-ground Gait Rehabilitation Robot Using Torque and Insole Pressure Sensors, Int. J. Control. Autom. Syst., vol. $16,275-283,2018$.

[13] A. Sarasola-Sanz, et al. A hybrid brain-machine interface based on EEG and EMG activity for the motor rehabilitation of stroke patients, en 2017 International Conference on Rehabilitation Robotics, 895-900, 2017.

[14] A. Qingsong, Y. Zhang, W. Qi, Q. Liu, y K. Chen. Research on lower limb motion recognition based on fusion of SEMG and accelerometer signals, Symmetry (Basel), vol. 9, 2017.

[15] O. Mazumder, A. S. Kundu, P. K. Lenka, y S. Bhaumik. Multi-channel Fusion Based Adaptive Gait Trajectory Generation Using Wearable Sensors, J. Intell. Robot. Syst. Theory Appl., vol. 86, 335-351, 2017. 
[16] V. Joukov, J. F.-S. Lin, y D. Kulic. Generalized Hebbian algorithm for wearable sensor rotation estimation, en 2017 IEEE/RSJ International Conference on Intelligent Robots and Systems, 2248-2253, 2017.

[17] S. J. Kim, Y. Kim, H. Lee, P. Ghasemlou, y J. Kim. Development of an MR-compatible hand exoskeleton that is capable of providing interactive robotic rehabilitation during $\mathrm{fMRI}$ imaging, Med. Biol. Eng. Comput., vol. 56, 261-272, 2018.

[18] S. Ferrante, et al. Neural and physiological measures to classify user's intention and control exoskeletons for rehabilitation or assistance: The experience@NearLab, 26th International Conference on Robotics, vol. 49, 735-745, 2018.

[19] A. M. Tobar, et al. Decoding of ankle flexion and extension from cortical current sources estimated from non-invasive brain activity recording methods, Front. Neurosci., vol. 11, 2018.

[20] H. Zeng-Guang, Z. Xin-Gang, C. Long, W. Qining, y W. Wei-qun. Recent Advances in Rehabilitation Robots and Intelligent Assistance Systems, vol. 15, 2016.

[21] A. Singla, S. Dhand, A. Dhawad and G. Virk. Toward Human-Powered Lower Limb Exoskeletons: A Review, Harmony Search and Nature Inspired Optimization Algorithms, 783-795, 2019.

[22] J. Bin Huang, K. Y. Young, y C. H. Ko. Effective control for an upper-body exoskeleton robot using ANFIS, 2016 IEEE Int. Conf. Syst. Sci. Eng, 2-5, 2016.

[23] A. M. Khan, F. Khan, y C. Han. Estimation of desired motion intention using extreme learning machine for upper limb assist exoskeleton, IEEE/ASME Int. Conf. Adv. Intell. Mechatronics, 919-923, 2016.

[24] Yupeng, K. Sang Hoon, P. Hyung-Soon, W. YiNing y Z. Li-Qun. Developing a multi-joint upper limb exoskeleton robot for diagnosis, therapy, and outcome evaluation in neurorehabilitation, IEEE Trans. Neural Syst. Rehabil. Eng., vol. 21, 490-499, 2013.
[25] U. Keller, H. van Hedel, V. KlamrothMarganska y R. Riener. ChARMin: The First Actuated Exoskeleton Robot for Pediatric Arm Rehabilitation, IEEE/ASME Trans. Mechatronics, vol. 21, 2201-2213, 2016.

[26] P. N. Kooren, et al. Design and control of the Active A-Gear: A wearable 5-DOF arm exoskeleton for adults with Duchenne muscular dystrophy, Proc. IEEE RAS EMBS Int. Conf. Biomed. Robot. Biomechatronics, vol. 2016, 637-642, 2016.

[27] J. Meuleman, E. van Asseldonk, G. van Oort, $\mathrm{H}$. Rietman y $\mathrm{H}$. van der Kooij. LOPES II Design and Evaluation of an Admittance Controlled Gait Training Robot with Shadow-Leg Approach, IEEE Transactions on Neural Systems and Rehabilitation Engineering, vol. 24, 352-363, 2016.

[28] G. Zeilig, et al. Lokomat walking results in increased metabolic markers in individuals with high spinal cord injury, Virtual Rehabil. Proc. (ICVR), 119-120, 2015.

[29] M. Mallwitz, N. Will, J. Teiwes, y E. A. Kirchner. The Capio Active Upper Body Exoskeleton and its Application For Teleoperation, Proc. 13th Symp. Adv. Sp. Technol. Robot. Autom., vol. 13, 1-8, 2015.

[30] A. Gardner, J. Potgieter y F. Noble. A review of commercially available exoskeletons' capabilities, 24th International Conference on Mechatronics and Machine Vision in Practice (M2VIP), 2017.

[31] A. Ruiz-Olaya, M. Callejas-Cuervo and C. LaraHerrera. Wearable low-cost inertial sensor-based electrogoniometer for measuring joint range of motion, DYNA 84 (201), 180-185, 2017.

[32] M. Callejas-Cuervo, R. Gutierrez y A. Hernandez. Joint amplitude MEMS based measurement platform for low cost and high accessibility telerehabilitation: Elbow case study, Journal of Bodywork and Movement Therapies, vol. 21, 574-581, 2017.

[33] A. Ruiz-Olaya, M. Callejas-Cuervo y A. Perez,. EMG-based pattern recognition with kinematics information for hand gesture recognition, 20th Symposium on Signal Processing, Images and Computer Vision (STSIVA), 2015. 